\title{
SOME WEAKLY MAPPINGS ON INTUITIONISTIC FUZZY TOPOLOGICAL SPACES
}

\author{
ZHEN-GUO XU AND FU-GUI SHI
}

\begin{abstract}
In this paper, we shall introduce concepts of fuzzy semiopen set, fuzzy semiclosed set, fuzzy semiinterior, fuzzy semiclosure on intuitionistic fuzzy topological space and fuzzy open (fuzzy closed) mapping, fuzzy irresolute mapping, fuzzy irresolute open (closed) mapping, fuzzy semicontinuous mapping and fuzzy semiopen (semiclosed) mapping between two intuitionistic fuzzy topological spaces. Moreover, we shall discuss their some properties.
\end{abstract}

\section{Introduction}

After the introduction of the concept of fuzzy sets by Zadeh in [8] several researches were conducted on the generalization of the notion of fuzzy set. The idea of "intuitionistic fuzzy set" was first published by Atanassov in [1] and many works by the same author and his colleagues appeared in the literature [2, 3, 4]. Later, this concept was generalized to "intuitionistic $L$-fuzzy sets" by Atanassov and Stoeva in [5]. In [7], the author introduced the concepts of intuitionistic fuzzy topological spaces and intuitionistic fuzzy continuous mappings.

In this paper, on base [7] we shall introduce the concepts of fuzzy semiopen set, fuzzy semiclosed set and fuzzy semiinterior and fuzzy semiclosure on intuitionistic fuzzy topological spaces and fuzzy irresolute mapping, fuzzy irresolute open (closed) mapping, fuzzy semicontinuous, fuzzy semiopen (semiclosed) mapping between two intuitionistic fuzzy topological spaces. Moreover, we shall discuss their some properties.

\section{Preliminaries}

Definition 2.1.([5]) Let $X$ be a nonempty set. An intuitionstic fuzzy set (IFS for short) $A$ is an object having the form

$$
A=\left\{\left\langle x, \mu_{A}(x), v_{A}(x)\right\rangle: x \in X\right\}
$$

where the mappings $\mu_{A}(x): X \rightarrow I$ and $v_{A}: X \rightarrow I$ denote the degree of membership and the degree of nonmembership of each element $x \in X$ to the set $A$, respectively and $0 \leq \mu_{A}(x)+$ $v_{A}(x) \leq 1$ for each $x \in X$.

Received March 09, 2006.

2000 Mathematics Subject Classification. 54A40.

Key words and phrases. Intuitionistic fuzzy set, intuitionistic fuzzy irresolute mapping, intuitionistic fuzzy semicontinuous mapping.

The project is supported by Scientific Research Common Program of Beijing Municipal Commission of Education (KM200510009009). 
Definition 2.2.([5]) Let $X$ be a nonempty set, the IFSs $A, B$ and $\left\{A_{i}, i \in I\right\}$ be in the form $A=\left\{\left\langle x, \mu_{A}(x), v_{A}(x)\right\rangle: x \in X\right\}, B=\left\{\left\langle x, \mu_{B}(x), v_{B}(x)\right\rangle: x \in X\right\}$ and $A_{i}=\left\{\left\langle x, \mu_{A_{i}}(x), v_{A_{i}}(x)\right\rangle: x \in\right.$ $X, i \in I\}$ be an arbitrary family of IFSs in $X$. Then

(1) $A \leq B$ if and only if $\mu_{A}(x) \leq \mu_{B}(x), v_{A}(x) \geq v_{B}(x)$ for each $x \in X$;

(2) $A^{\prime}=\left\{\left\langle x, v_{A}(x), \mu_{A}(x)\right\rangle: x \in X\right\}$;

(3) $\wedge A_{i}=\left\{\left\langle x, \wedge \mu_{A_{i}}(x), \bigvee v_{A_{i}}(x)\right\rangle: x \in X\right\}$;

(4) $\bigvee_{i \in I}^{i \in I} A_{i}=\left\{\left\langle x, \bigvee_{i \in I} \mu_{A_{i}}(x), \bigwedge_{i \in I}^{i \in I} v_{A_{i}}(x)\right\rangle: x \in X\right\}$

Definition 2.3.([7]) $\underline{0}=\{\langle x, 0,1\rangle: x \in X\}$ and $\underline{1}=\{\langle x, 1,0\rangle: x \in X\}$.

Proposition 2.4.([7]) Let $A, B, C$ be IFSs in $X$. Then

(1) $(A \vee B)^{\prime}=A^{\prime} \wedge B^{\prime},(A \wedge B)^{\prime}=A^{\prime} \vee B^{\prime}$;

(2) $A \leq B \Rightarrow B^{\prime} \leq A^{\prime}$;

(3) $\left(A^{\prime}\right)^{\prime}=A, \underline{1}^{\prime}=\underline{0}, \underline{0}^{\prime}=\underline{1}$.

By above some definitions, we have the following theorem:

Theorem 2.5.([7]) Let $A, A_{i}(i \in I)$ be IFSs in $X, B, B_{j}(j \in J)$ be IFSs in $Y$ and $f: X \rightarrow Y$ be a mapping as defined in [7]. Then

(1) $A_{1} \leq A_{2} \Rightarrow f_{L}^{\rightarrow}\left(A_{1}\right) \leq f_{L}^{\rightarrow}\left(A_{2}\right), B_{1} \leq B_{2} \Rightarrow f_{L}^{\leftarrow}\left(B_{1}\right) \leq f_{L}^{\leftarrow}\left(B_{2}\right)$;

(2) $A \leq f_{L}^{\leftarrow}\left(f_{L}^{-}(A)\right), f_{L}^{\rightarrow}\left(f_{L}^{\leftarrow}(B)\right) \leq B$

(3) $f_{L}^{\leftarrow}\left(B^{\prime}\right)=\left(f_{L}^{\leftarrow}(B)\right)^{\prime}$.

Definition 2.6.([7]) An intuitionistic fuzzy topological space (IFTS for short) is a pair $(X, \tau)$, where $\tau$ is a subfamily of IFSs in $X$ which contains $\underline{0}, \underline{1}$ and is closed for any suprema and finite infima. $\tau$ is called an intuitionistic fuzzy topology on $X$. Each member of $\tau$ is called an intuitionistic open set (IFCS for short) and its quasi-complementation is called an intuitionistic closed set(IFCS for short).

Definition 2.7.([7]) Let $(X, \tau)$ be an IFTS and $A=\left\{\left\langle x, \mu_{A}(x), v_{A}(x)\right\rangle: x \in X\right\}$ be an IFS in $X$. Then the fuzzy interior and fuzzy closure of $A$ are defined by

$\operatorname{cl}(A)=\bigwedge\{C: C$ is an IFCS in $X$ and $C \geq A\}$;

$\operatorname{int}(A)=\bigvee\{D: D$ is an IFOS in $X$ and $D \leq A\}$.

It can be also shown that $\operatorname{cl}(A)$ is an IFCS, $\operatorname{int}(A)$ is an IFOS in $X$ and $A$ is an IFCS in $X$ if and only if $\operatorname{cl}(A)=A$; $A$ is an IFOS in $X$ if and only if $\operatorname{int}(A)=A$.

Proposition 2.8.([7]) Let $(X, \tau)$ be an IFTS and $A, B$ be IFSs in $X$. Then the following properties hold:

(1) $\operatorname{cl}\left(A^{\prime}\right)=(\operatorname{int}(A))^{\prime}, \operatorname{int}\left(A^{\prime}\right)=(\operatorname{cl}(A))^{\prime}$;

(2) $\operatorname{int}(A) \leq A \leq \operatorname{cl}(A)$;

(3) if $A \leq B$, then int $(A) \leq \operatorname{int}(B), \operatorname{cl}(A) \leq \operatorname{cl}(B)$.

Definition 2.9.([7]) Let $(X, \tau),(Y, \sigma)$ be two IFTSs and let $f: X \rightarrow Y$ be a mapping. Then $f$ is said to be fuzzy continuous if and only if the primage of each IFS in $\sigma$ is an IFS in $\tau$. 


\section{Intuitionistic fuzzy semiopen (semiclosed) set and some weakly mappings}

Definition 3.1. Let $(X, \tau)$ be an IFTS and $A=\left\{\left\langle x, \mu_{A}(x), v_{A}(x)\right\rangle: x \in X\right\}$ be an IFS in $X$. Then $A$ is called:

(1) fuzzy semiopen set (IFSOS for short) if and only if there exists a $B \in \tau$ such that $B \leq A \leq$ $\operatorname{cl}(B)$;

(2) fuzzy semiclosed set (IFSCS for short) if and only if there exists a $B^{\prime} \in \tau$ such that $i n t(B) \leq$ $A \leq B$

(3) $\operatorname{sint}(A)=\bigvee\{B: B$ is an IFSOS and $B \leq A\}$;

(4) $\operatorname{scl}(A)=\bigwedge\{C: C$ is an IFSCS and $A \leq C\}$.

It can be also shown that $\operatorname{scl}(A)$ is an IFSCS and $\operatorname{sint}(A)$ is an IFSOS in $X$

Corollary 3.2. Let $(X, \tau)$ be an IFTS and $A=\left\{\left\langle x, \mu_{A}(x), v_{A}(x)\right\rangle: x \in X\right\}$ be an IFS in $X$. Then $A$ is an IFSOS if and only if $A \leq \operatorname{cl}(\operatorname{int}(A))$.

Proof. Let $A$ be an IFSOS. Then there exists a $B \in \tau$ such that $B \leq A \leq c l B$, by Definition 2.7 and Proposition 2.8 follows that $B \leq A \leq \operatorname{cl} B=\operatorname{cl}(\operatorname{int}(B)) \leq \operatorname{cl}(\operatorname{int}(A)))$, i.e, $A \leq \operatorname{cl}(\operatorname{int}(A))$.

Conversely, let $A \leq \operatorname{cl}(\operatorname{int}(A))$. Then $\operatorname{int}(A) \leq A \leq \operatorname{cl}(\operatorname{int}(A))$, let $B=\operatorname{int}(A)$, thus there exists a $B \in \tau$ such that $B \leq A \leq \operatorname{cl}(B)$. Hence $A$ is an IFSOS.

Remark 3.3. From Definition 3.1, we can know that IFOS (IFCS) is IFSOS (IFSCS), but the inverses is false is shown by the following Example 3.4 .

Example 3.4. Let $X=\{a, b\}$ and $A=\left\{\left\langle x,\left(\frac{a}{0.3}, \frac{b}{0.4}\right),\left(\frac{a}{0.3}, \frac{b}{0.5}\right)\right\rangle: x \in X\right\}$.

Then the family $\tau=\{\underline{0}, \underline{1}, A\}$ of IFSs in $X$ is an IFT on $X$. Let

$$
C=\left\{\left\langle x,\left(\frac{a}{0.2}, \frac{b}{0.4}\right),\left(\frac{a}{0.6}, \frac{b}{0.6}\right)\right\rangle: x \in X\right\} .
$$

Then $C$ is not an IFOS, but

$$
\operatorname{cl}(\operatorname{int}(C))=\left\{\left\langle x,\left(\frac{a}{0.3}, \frac{b}{0.5}\right),\left(\frac{a}{0.3}, \frac{b}{0.4}\right)\right\rangle: x \in X\right\},
$$

hence $C \leq \operatorname{cl}(\operatorname{int}(C))$, i.e, $C$ is an IFSOS.

By Definition 3.1, we have the following theorem.

Theorem 3.5. Let $(X, \tau)$ be an IFTS and $A=\left\{\left\langle x, \mu_{A}(x), v_{A}(x)\right\rangle: x \in X\right\}$ be an IFS in $X$. Then

(1) $A$ is an IFSOS if and only if $A=\operatorname{sint}(A)$;

(2) $A$ is an IFSCS if and only if $A=\operatorname{scl}(A)$;

(3) $\underline{0}=\operatorname{scl}(\underline{0}), \underline{1}=\operatorname{sint}(\underline{1})$;

(4) $\operatorname{sint}(A)=\left(\operatorname{scl}\left(A^{\prime}\right)\right)^{\prime}$;

(5) $\operatorname{scl}(\operatorname{scl}(A))=\operatorname{scl}(A), \operatorname{sint}(\operatorname{sint}(A))=\operatorname{sint}(A)$;

(6) $\operatorname{scl}(\operatorname{cl}(A))=\operatorname{cl}(A), \operatorname{sint}(\operatorname{int}(A))=\operatorname{int}(A)$; 
(7) If $A$ is an IFSOS (IFSCS), then int $(\operatorname{sint}(A))=\operatorname{int}(A)(\operatorname{cl}(\operatorname{scl}(A))=\operatorname{cl}(A))$.

Using IFOS, IFCS, IFSOS and IFSCS we can obtain the following definitions:

Definition 3.6. Let $(X, \tau),(Y, \sigma)$ be two IFTSs and let $f: X \rightarrow Y$ be a mapping. Then $f$ is said to be fuzzy semicontinuous if and only if the preimage of each IFS in $\sigma$ is an IFSOS in $X$.

Definition 3.7. Let $(X, \tau),(Y, \sigma)$ be two IFTSs and let $f: X \rightarrow Y$ be a mapping. Then $f$ is said to be fuzzy open (closed) if and only if the image of each IFS in $\tau\left(\tau^{\prime}\right)$ is an IFS in $\sigma\left(\sigma^{\prime}\right)$.

Definition 3.8. Let $(X, \tau),(Y, \sigma)$ be two IFTSs and let $f: X \rightarrow Y$ be a mapping. Then $f$ is said to be fuzzy semiopen (semiclosed) if and only if the image of each IFS in $\tau$ is an IFSOS(IFSCS) in $Y$.

Definition 3.9. Let $(X, \tau),(Y, \sigma)$ be two IFTSs and let $f: X \rightarrow Y$ be a mapping. Then $f$ is said to be fuzzy irresolute if and only if the preimage of each IFSOS in $Y$ is an IFSOS in $X$.

Definition 3.10. Let $(X, \tau),(Y, \sigma)$ be two IFTSs and let $f: X \rightarrow Y$ be a mapping. Then $f$ is said to be fuzzy irresolute open (closed) if and only if the image of each IFSOS (IFSCS) in $X$ is an IFSOS (IFSCS) in $Y$.

By Definition 2.9, Definitions 3.6-3.10, we can obtain following relations:

Theorem 3.11. Let $(X, \tau),(Y, \sigma)$ be two IFTSs and let $f: X \rightarrow Y$ be a mapping.

(1) If $f$ is fuzzy continuous, then $f$ is fuzzy semicontinuous;

(2) If $f$ is fuzzy open (closed), then $f$ is fuzzy semiopen (semiclosed);

(3) If $f$ is fuzzy irresolute, then $f$ is fuzzy semicontinuous.

Remark 3.12. The inverse of Theorem 3.11 is not true. This can be seen from the following examples.

Example 3.13. Let $X=\{a, b\}, Y=\{c, d\}$ and

$$
\begin{aligned}
& A=\left\{\left\langle x,\left(\frac{a}{0.3}, \frac{b}{0.4}\right),\left(\frac{a}{0.6}, \frac{b}{0.5}\right)\right\rangle: x \in X\right\}, \\
& B=\left\{\left\langle y,\left(\frac{c}{0.3}, \frac{d}{0.4}\right),\left(\frac{c}{0.3}, \frac{d}{0.5}\right)\right\rangle: y \in Y\right\} .
\end{aligned}
$$

Let $(X, \tau)$ and $(Y, \sigma)$ be two IFTSs, where $\tau=\{\underline{0}, \underline{1}, A\}$ and $\sigma=\{\underline{0}, \underline{1}, B\} . f:(X, \tau) \rightarrow(Y, \sigma)$ defined by $f(a)=c, f(b)=d$ is not continuous, because $f^{\leftarrow}(B)=\left\{\left\langle x,\left(\frac{a}{0.3}, \frac{b}{0.4}\right),\left(\frac{a}{0.3}, \frac{b}{0.5}\right)\right\rangle: x \in\right.$ $X\}$ is not an IFOS in $X$, but

$$
\operatorname{cl}\left(\operatorname{int}\left(f_{L}^{\leftarrow}(B)\right)\right)=\left\{\left\langle x,\left(\frac{a}{0.6}, \frac{b}{0.5}\right),\left(\frac{a}{0.3}, \frac{b}{0.4}\right)\right\rangle: x \in X\right\} .
$$

Hence $f_{L}^{\leftarrow}(B) \leq \operatorname{cl}\left(\operatorname{int}\left(f_{L}^{\leftarrow}(B)\right)\right)$, i.e, $f_{L}^{\leftarrow}(B)$ is an IFSOS in $X$. Therefore $f$ is semicontinuous.

Example 3.14. Let $X=\{a, b\}, Y=\{c, d\}$ and

$$
A=\left\{\left\langle x,\left(\frac{a}{0.6}, \frac{b}{0.5}\right),\left(\frac{a}{0.4}, \frac{b}{0.4}\right)\right\rangle: x \in X\right\}, B=\left\{\left\langle y,\left(\frac{c}{0.2}, \frac{d}{0.4}\right),\left(\frac{c}{0.6}, \frac{d}{0.5}\right)\right\rangle: y \in Y\right\} .
$$


Let $(X, \tau)$ and $(Y, \sigma)$ be two IFTSs, where $\tau=\{\underline{0}, \underline{1}, A\}$ and $\sigma=\{\underline{0}, \underline{1}, B\} . f:(X, \tau) \rightarrow(Y, \sigma)$ defined by $f(a)=c, f(b)=d$ is semicontinuous, but it is not irresolute. In fact, let $C=$ $\left\{\left\langle y,\left(\frac{c}{0.5}, \frac{d}{0.4}\right),\left(\frac{c}{0.5}, \frac{d}{0.5}\right)\right\rangle: y \in Y\right\}$ in $Y$. Then

$$
\operatorname{cl}(\operatorname{int}(C))=\left\{\left\langle y,\left(\frac{c}{0.6}, \frac{d}{0.5}\right),\left(\frac{c}{0.2}, \frac{d}{0.4}\right)\right\rangle: y \in Y\right\},
$$

thus $C$ is an IFSOS. Moreover, we know $f_{L}^{\leftarrow}(C)=\left\{\left\langle x,\left(\frac{a}{0.5}, \frac{b}{0.4}\right),\left(\frac{a}{0.5}, \frac{b}{0.5}\right)\right\rangle: x \in X\right\}$, hence

$$
\operatorname{cl}\left(\operatorname{int}\left(f_{L}^{\leftarrow}(C)\right)\right)=\left\{\left\langle x,\left(\frac{a}{0.4}, \frac{b}{0.4}\right),\left(\frac{a}{0.6}, \frac{b}{0.5}\right)\right\rangle: x \in X\right\},
$$

i.e, $f_{L}^{\leftarrow}(C) \not \subset \operatorname{cl}\left(\operatorname{int}\left(f_{L}^{\leftarrow}(C)\right)\right.$, therefore $f$ is not irresolute.

From definition of $f$, we obtain $f_{L}^{\rightarrow}(A)=\left\{\left\langle y,\left(\frac{c}{0.6}, \frac{d}{0.5}\right),\left(\frac{c}{0.4}, \frac{d}{0.4}\right)\right\rangle: y \in Y\right\}$, thus $f_{L}^{\rightarrow}(A) \neq B$, i.e, $f$ is not a fuzzy open mapping, but

$$
\operatorname{cl}\left(\operatorname{int}\left(f_{L}^{\rightarrow}(A)\right)\right)=\left\{\left\langle y,\left(\frac{c}{0.6}, \frac{d}{0.5}\right),\left(\frac{c}{0.2}, \frac{d}{0.4}\right)\right\rangle: y \in Y\right\},
$$

then $f_{L}^{\rightarrow}(A) \leq \operatorname{cl}\left(\operatorname{int}\left(f_{L}^{\rightarrow}(A)\right)\right)$, therefore $f_{L}^{\rightarrow}(A)$ is a fuzzy semiopen set in $Y$, hence $f$ is a semiopen mapping.

\section{The properties of some weakly mappings}

Theorem 4.1. Let $(X, \tau),(Y, \sigma)$ be two IFTSs and let $f: X \rightarrow Y$ be a mapping. Then the following conditions are equivalent.

(1) $f$ is fuzzy irresolute;

(2) $f_{L}^{\leftarrow}(B)$ is an IFSCS in $X$ for each IFSCS $B$ in $Y$;

(3) $f_{L}^{\rightarrow}(\operatorname{scl}(A)) \leq \operatorname{scl}\left(f_{L}^{\rightarrow}(A)\right)$ for each IFS $A$ in $X$;

(4) $\operatorname{scl}\left(f_{L}^{\leftarrow}(B)\right) \leq f_{L}^{\leftarrow}(\operatorname{scl}(B))$ for each IFS $B$ in $Y$;

(5) $f_{L}^{\leftarrow}(\operatorname{sint}(B)) \leq \operatorname{int}\left(f_{L}^{\leftarrow}(B)\right)$ for each IFS $B$ in $Y$.

Proof. (1) $\Rightarrow(2)$ is obvious.

(2) $\Rightarrow(3)$. For any IFS $A$ in $X$, we have $A \leq f_{L}^{\leftarrow}\left(f_{L}^{\rightarrow}(A)\right) \leq f_{L}^{\leftarrow}\left(\operatorname{scl}\left(f_{L}^{-}(A)\right)\right)$, we know that $\operatorname{scl}\left(f_{L}^{\rightarrow}(A)\right)$ is an IFSCS in $Y$ from Definition 3.1, hence by (2), $f_{L}^{\leftarrow}\left(\operatorname{scl}\left(f_{L}^{-}(A)\right)\right)$ is an IFSCS in $X$, thus by Definition 3.1(4) we obtain $\operatorname{scl}(A) \leq f_{L}^{\leftarrow}\left(\operatorname{scl}\left(f_{L}^{\rightarrow}(A)\right)\right)$, therefore

$$
f_{L}^{\rightarrow}(\operatorname{scl}(A)) \leq f_{L}^{\rightarrow}\left(f_{L}^{\leftarrow}\left(\operatorname{scl}\left(f_{L}^{\rightarrow}(A)\right)\right)\right) \leq \operatorname{scl}\left(f_{L}^{\rightarrow}(A)\right) .
$$

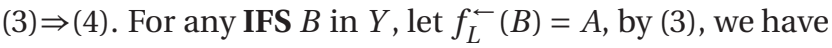

$$
f_{L}^{\rightarrow}\left(\operatorname{scl}\left(f_{L}^{\leftarrow}(B)\right) \leq \operatorname{scl}\left(f_{L}^{\rightarrow}\left(f_{L}^{\leftarrow}(B)\right)\right) \leq \operatorname{scl}(B),\right.
$$

this implies

$$
\operatorname{scl}\left(f_{L}^{\leftarrow}(B) \leq f_{L}^{\leftarrow}\left(f_{L}^{-}\left(\operatorname{scl}\left(f_{L}^{\leftarrow}(B)\right)\right)\right) \leq f_{L}^{\leftarrow}(\operatorname{scl}(B)) .\right.
$$


(4) $\Rightarrow(5)$. For any IFS $B$ in $Y$, by $\operatorname{sint}(B)=\left(\operatorname{scl}\left(B^{\prime}\right)\right)^{\prime}$ and (4), we have

$$
\begin{aligned}
f_{L}^{\leftarrow}(\operatorname{sint}(B)) & =f_{L}^{\leftarrow}\left(\left(\operatorname{scl}\left(B^{\prime}\right)\right)^{\prime}\right) \\
& =\left(f_{L}^{\leftarrow}\left(\operatorname{scl}\left(B^{\prime}\right)\right)\right)^{\prime} \\
& \leq\left(\operatorname{scl}\left(f_{L}^{\leftarrow}\left(B^{\prime}\right)\right)\right)^{\prime} \\
& =\left(\left(\operatorname{sint}\left(f_{L}^{\leftarrow}(B)\right)\right)^{\prime}\right)^{\prime} \\
& =\operatorname{sint}\left(f_{L}^{\leftarrow}(B)\right)
\end{aligned}
$$

(5) $\Rightarrow(1)$. Let $B$ be an IFSOS in $Y$, then $B=\operatorname{sint}(B)$ from Theorem 3.5, by (5) we obtain $f_{L}^{\leftarrow}(B) \leq \operatorname{sint}\left(f_{L}^{\leftarrow}(B)\right)$. On the other hand $f_{L}^{\leftarrow}(B) \geq \operatorname{sint}\left(f_{L}^{\leftarrow}(B)\right)$ by Definition 3.1, thus $f_{L}^{\leftarrow}(B)=\operatorname{sint}\left(f_{L}^{\leftarrow}(B)\right)$, therefore $f_{L}^{\leftarrow}(B)$ is an IFSOS in $X$ from Theorem 3.5.

Theorem 4.2. Let $(X, \tau),(Y, \sigma)$ be two IFTSs and let $f: X \rightarrow Y$ be a mapping. Then the following conditions are equivalent.

(1) $f$ is fuzzy semicontinuous;

(2) $f_{L}^{\leftarrow}(B)$ is an IFSCS in $X$ for each IFCS $B$ in $Y$;

(3) $\operatorname{scl}\left(f_{L}^{\leftarrow}(B)\right) \leq f_{L}^{\leftarrow}(\operatorname{cl}(B))$ for each IFS $B$ in $Y$;

(4) $f_{L}^{\leftarrow}(\operatorname{int}(B)) \leq \operatorname{sint}\left(f_{L}^{\leftarrow}(B)\right)$ for each IFS $B$ in $Y$;

(5) $f_{L}^{-}(B) \leq c l\left(\operatorname{sint}\left(f_{L}^{-}(B)\right)\right)$ for each IFOS $B$ in $Y$.

Proof. (1) $\Rightarrow(2)$ is obvious.

$(2) \Rightarrow(3)$. Let $B$ be IFS in $Y$. Then $c l(B)$ is an IFCS, so by (2), $f_{L}^{\leftarrow}(c l(B))$ is an IFSCS in $X$. Noting that $B \leq c l(B)$, we obtain $f_{L}^{\leftarrow}(B) \leq f_{L}^{\leftarrow}(c l(B))$, hence $\operatorname{scl}\left(f_{L}^{\leftarrow}(B)\right) \leq f_{L}^{\leftarrow}(c l(B))$ from Definition 3.1.

(3) $\Rightarrow(4)$. This proof is easily and therefore omitted.

$(4) \Rightarrow(5)$. For any IFOS $B$ in $Y$. Then $B=\operatorname{int}(B)$, thus $f_{L}^{\leftarrow}(B)=f_{L}^{\leftarrow}(\operatorname{int}(B)) \leq \operatorname{sint}\left(f_{L}^{\leftarrow}(B)\right)$, hence $f_{L}^{\leftarrow}(B)=\operatorname{sint}\left(f_{L}^{\leftarrow}(B)\right)$, therefore $f_{L}^{\leftarrow}(B)$ is an IFSOS from Theorem 3.5. Thus $f_{L}^{\leftarrow}(B) \leq$ $\operatorname{cl}\left(\operatorname{int}\left(f_{L}^{-}(B)\right)\right)$ from Corollary 3.2.

$(5) \Rightarrow(1)$. It follows immediately from Corollary 3.2 and therefore omitted.

Theorem 4.3. Let $(X, \tau),(Y, \sigma)$ be two IFTSs and let $f: X \rightarrow Y$ be a mapping. Then the following conditions are equivalent.

(1) $f$ is fuzzy irresolute open;

(2) $f_{L}^{\rightarrow}(\operatorname{sint}(A)) \leq \operatorname{sint}\left(f_{L}^{\rightarrow}(A)\right)$ for each IFS $A$ in $X$;

(3) $\operatorname{sint}\left(f_{L}^{\leftarrow}(B)\right) \leq f_{L}^{\leftarrow}(\sin t(B))$ for each IFS $B$ in $Y$;

(4) For any IFS $A$ in $X$, IFS $B$ in $Y$ and let $A$ be the IFSCS such that $f_{L}^{\leftarrow}(B) \leq A$. Then there exists an IFSCS $C$ in $Y$ and $B \leq C$ such that $f_{L}^{-}(C) \leq A$.

Proof. $(1) \Rightarrow(2)$. By Definition 3.1(3), we have $\operatorname{sint}(A) \leq A$, hence $f_{L}^{\rightarrow}(\operatorname{sint}(A)) \leq f_{L}(A)$ and by Definition 3.1, we know $\operatorname{sint}(A)$ is an IFSOS in $X$, thus $f_{L}^{\rightarrow}(\operatorname{sint}(A)) \leq \operatorname{sint}\left(f_{L}^{\rightarrow}(A)\right)$.

(2) $\Rightarrow(3)$. Let $A=f_{L}^{\leftarrow}(B)$. Form (2) we have

$$
f_{L}^{\rightarrow}\left(\operatorname{sint}\left(f_{L}^{\leftarrow}(B)\right)\right) \leq \operatorname{sint}\left(f_{L}^{\rightarrow}\left(f_{L}^{\leftarrow}(B)\right)\right) \leq \operatorname{sint}(B),
$$

this implies

$$
\operatorname{sint}\left(f_{L}^{\leftarrow}(B)\right) \leq f_{L}^{\leftarrow}\left(f_{L}^{\rightarrow}\left(\operatorname{sint}\left(f_{L}^{\leftarrow}(B)\right)\right)\right) \leq f_{L}^{\leftarrow}(\operatorname{sint}(B)),
$$


i.e,

$$
\operatorname{sint}\left(f_{L}^{\leftarrow}(B)\right) \leq f_{L}^{\leftarrow}(\operatorname{sint}(B)) .
$$

$(3) \Rightarrow(4)$. Let $A$ be an IFSCS in $X$ and $B$ be an IFS in $Y$ such that $f_{L}^{-}(B) \leq A$, hence $A^{\prime} \leq$ $f_{L}^{\leftarrow}\left(B^{\prime}\right)$, we know that $A^{\prime}$ is an IFSOS, thus $\operatorname{sint}\left(A^{\prime}\right)=A^{\prime} \leq \operatorname{sint}\left(f_{L}^{\leftarrow}\left(B^{\prime}\right)\right)$ form Proposition 2.8, therefore $A^{\prime} \leq \operatorname{sint}\left(f_{L}^{\leftarrow}\left(B^{\prime}\right)\right) \leq f_{L}^{\leftarrow}\left(\operatorname{sint}\left(B^{\prime}\right)\right)$, this implies $A \geq\left(f_{L}^{\leftarrow}\left(\operatorname{sint}\left(B^{\prime}\right)\right)\right)^{\prime}=f_{L}^{\leftarrow}(\operatorname{scl}(B))$, let $C=\operatorname{scl}(B)$, then $C$ satisfies condition of (4).

(4) $\Rightarrow(1)$. Let $D$ be an IFSOS in $X, B=\left(f_{L}^{\rightarrow}(D)\right)^{\prime}, A=D^{\prime}$. Then $A$ is an IFSCS, hence $f_{L}^{\leftarrow}(B)=$ $f_{L}^{\leftarrow}\left(\left(f_{L}^{\rightarrow}(D)\right)^{\prime}\right)=\left(f_{L}^{\leftarrow}\left(f_{L}^{\rightarrow}(D)\right)\right)^{\prime} \leq D^{\prime}=A$, by (4), there exists an IFSCS $C$ and $B \leq C$ such that $f_{L}^{\leftarrow}(C) \leq A=D^{\prime}$, thus $D \leq\left(f_{L}^{\leftarrow}(C)\right)^{\prime}$, thus $f_{L}^{\rightarrow}(D) \leq f_{L}^{\rightarrow}\left(f_{L}^{\leftarrow}\left(C^{\prime}\right)\right) \leq C^{\prime}$. On the other hand by $B \leq C, f_{L}^{\rightarrow}(D)=B^{\prime} \geq C^{\prime}$, hence $f_{L}^{\overrightarrow{ }}(D)=C^{\prime}$. Since $C^{\prime}$ is an IFSOS, we have $f_{L}^{\rightarrow}(D)$ is an IFSOS.

Analogously, we can prove following theorems:

Theorem 4.4. Let $(X, \tau),(Y, \sigma)$ be two IFTSs and let $f: X \rightarrow Y$ be a mapping. Then the following conditions are equivalent.

(1) $f$ is fuzzy irresolute closed;

(2) $f_{L}^{\rightarrow}\left(\operatorname{scl}(A) \geq \operatorname{scl}\left(f_{L}^{\rightarrow}(A)\right)\right.$ for each IFS $A$ in $X$;

(3) $\operatorname{scl}\left(f_{L}^{\leftarrow}(B)\right) \geq f_{L}^{\leftarrow}(\operatorname{scl}(A))$ for each IFS $B$ in $Y$;

(4) For any IFS $A$ in $X$ and IFS $B$ in $Y$, let $A$ be the IFSOS such that $f_{L}^{\leftarrow}(B) \leq A$. Then there exists an IFSOS $C$ in $Y$ and $B \leq C$ such that $f_{L}^{\leftarrow}(C) \leq A$.

Theorem 4.5. Let $(X, \tau),(Y, \sigma)$ be two IFTSs and let $f: X \rightarrow Y$ be a mapping. Then the following conditions are equivalent.

(1) $f$ is fuzzy semiopen;

(2) $f_{L}^{\rightarrow}\left(\operatorname{int}(A) \leq \operatorname{sint}\left(f_{L}^{\rightarrow}(A)\right)\right.$ for each IFS $A$ in $X$;

(3) $\operatorname{int}\left(f_{L}^{\leftarrow}(B)\right) \leq f_{L}^{\leftarrow}(\operatorname{sint}(B))$ for each IFS $B$ in $Y$.

Theorem 4.6. Let $(X, \tau)$ and $(Y, \sigma)$ be two IFTSs and let $f: X \rightarrow Y$ be a mapping. Then the following conditions are equivalent.

(1) $f$ is fuzzy semiclosed;

(2) $\operatorname{int}\left(\operatorname{cl}\left(f_{L}^{\rightarrow}(A)\right)\right) \geq f_{L}^{\rightarrow}(\operatorname{cl}(A))$ for each IFS $A$ in $X$;

(3) $\operatorname{scl}\left(f_{L}^{\rightarrow}(A)\right) \leq f_{L}^{\rightarrow}(\operatorname{cl}(A))$ for each IFS $A$ in $X$.

\section{References}

[1] K. Atanassov and S. Stoeva, Intuitionistic fuzzy sets, in Polish Svmp on Interval and Fuzzy Mathematics, Poznan (August 1983) 23-26.

[2] K. Atanassov, Intuitionistic fuzzy sets, in: V. Sgurev, Ed., VIIITKR's Session. Sofia (June 1983 Central Sci. and Techn. Library, Bulg. Academy of Sciences, 1984).

[3] K. Atanassov adn S. Stoeva, Intuitionistic L-fuzzy sets, in: R.Trappl, Ed., Cybernetics and Systems Research, Vol. 2(Elsevier, Amsterdam, 1984), 539-540.

[4] K. Atanassov, Intuitionistic fuzzy sets, Fuzzy Sets and Systems 20(1986), 87-96.

[5] K. Atanassov, Review and new results on intuitionistic fuzzy sets, Preprint IM-MFAIS-1-88, Sofia, 1988. 
[6] K. K. Azad, On Fuzzy semicontinuity, fuzzy almost continuity and fuzzy weakly continuity, J. Math. Anal. Appl., 82(1981), 14-32.

[7] Doğan Coker, An introduction to tinuitionistic fuzzy topological spaces, Fuzzy Sets and Systems 88(1997), 81-89.

[8] L. A. Zadeh, Fuzzy sets, Inform. and Control, 8(1965), 338-353.

Department of Mathematics, Beijing Institute of Technology, Beijing 100081, P.R. China.

E-mail: zhenguoxu@126.com

E-mail: fuguishi@bit.edu.cn 